PROCEEDINGS OF THE

AMERICAN MATHEMATICAL SOCIETY

Volume 132, Number 7, Pages 1957-1962

S 0002-9939(04)06985-0

Article electronically published on February 26, 2004

\title{
REFLECTION SYMMETRIES AND ABSENCE OF EIGENVALUES FOR ONE-DIMENSIONAL SCHRÖDINGER OPERATORS
}

\author{
DAVID DAMANIK AND DIRK HUNDERTMARK
}

(Communicated by Joseph A. Ball)

\begin{abstract}
We prove a criterion for absence of decaying solutions for onedimensional Schrödinger operators. As necessary input, we require infinitely many centers of local reflection symmetry and upper and lower bounds for the traces of the associated transfer matrices.
\end{abstract}

\section{INTRODUCTION}

In this article we are interested in the spectral properties of one-dimensional Schrödinger operators on the half line,

$$
H_{\theta}=-\frac{d^{2}}{d x^{2}}+V(x) \text { on } L^{2}(0, \infty)
$$

with boundary condition

$$
\psi(0) \cos (\theta)+\psi^{\prime}(0) \sin (\theta)=0
$$

$\theta \in(-\pi / 2, \pi / 2]$, or on the whole line,

$$
H=-\frac{d^{2}}{d x^{2}}+V(x) \text { on } L^{2}(\mathbb{R})
$$

together with the associated eigenvalue equation

$$
-\phi^{\prime \prime}(x)+V(x) \phi(x)=E \phi(x),
$$

where we assume $V$ to be locally integrable.

We will be interested in criteria excluding the existence of decaying solutions to (4) that are based on local reflection symmetries. Our approach is similar in spirit to the one by Jitomirskaya and Simon [3], but their criterion only works for whole-line models and does not exclude the existence of decaying solutions, but merely the existence of $L^{2}$-solutions. On the other hand, apart from local reflection symmetries we need to require further assumptions, namely, suitable bounds on transfer matrix traces.

To this end, we work out the continuum counterpart to the recent work [2] where a criterion in this direction was established for discrete one-dimensional Schrödinger

Received by the editors July 3, 2002 and, in revised form, August 1, 2002.

2000 Mathematics Subject Classification. Primary 34L05, 47E05, 81Q10.

Key words and phrases. Schrödinger operators, eigenvalue problem, local reflection symmetries.

Supported in part by NSF grant DMS-0010101. 
operators. This transition is, in fact, not straightforward since the transfer matrices in the continuum case do not have a certain form which was crucial in the proof of [2]. We refer to [1] and [2] for more details on the history of criteria employing local symmetries for excluding decaying solutions to the Schrödinger equation. While we focus here on proving the continuum analogue to the abstract criterion from [2], we refer the reader to [2] for applications and illustrations of this criterion. The necessary input, bounds on transfer matrix traces, can typically be established for self-similar potentials which are generated by a substitution rule. The trace bounds follow from the self-similarity of the potential and the unimodularity of the transfer matrices and are therefore rather model-independent.

Recall the notion of transfer matrix, which is a standard tool in the spectral theory of one-dimensional Schrödinger operators: For every $E \in \mathbb{R}$ and $x, y \in$ $[0, \infty)$, there is a $(2 \times 2)$-matrix $M_{E}(x, y)$ such that for every solution $\phi$ to (4), we have

$$
\left(\begin{array}{c}
\phi(x) \\
\phi^{\prime}(x)
\end{array}\right)=M_{E}(x, y)\left(\begin{array}{c}
\phi(y) \\
\phi^{\prime}(y)
\end{array}\right) .
$$

The matrix $M_{E}(x, y)$ is given by

$$
M_{E}(x, y)=\left(\begin{array}{ll}
\phi_{N}(x) & \phi_{D}(x) \\
\phi_{N}^{\prime}(x) & \phi_{D}^{\prime}(x)
\end{array}\right),
$$

where $\phi_{N}, \phi_{D}$ are the solutions to (44) obeying $\phi_{N}(y)=\phi_{D}^{\prime}(y)=1$ and $\phi_{N}^{\prime}(y)=$ $\phi_{D}(y)=0$. We shall write $M_{E}(x)$ for $M_{E}(x, 0)$.

We can now formulate our main result:

Theorem 1. Fix some $E \in \mathbb{R}$. Suppose that

(i) there exists a sequence $x_{k} \rightarrow \infty$ such that for every $k$ and every $0 \leq x \leq$ $x_{k} / 2$, we have $V(x)=V\left(x_{k}-x\right)$;

(ii) the sequence $\left(\left|\operatorname{tr} M_{E}\left(x_{k}\right)\right|\right)_{k \in \mathbb{N}}$ does not diverge to $\infty$;

(iii) the sequence $\left(\left|\operatorname{tr} M_{E}\left(x_{k}\right)\right|\right)_{k \in \mathbb{N}}$ does not converge to 0 .

Then for every nontrivial solution $\phi$ to (4), we have $|\phi(x)|^{2}+\left|\phi^{\prime}(x)\right|^{2} \nrightarrow 0$ as $x \rightarrow \infty$.

Using Harnack's inequality (see 4]), we deduce the absence of $L^{2}$-solutions and hence obtain the following corollaries:

Corollary 1 (Half-line case). Under the assumptions of Theorem 1, we have that for every $\theta, E$ is not an eigenvalue of $H_{\theta}$.

Corollary 2 (Whole-line case). Under the assumptions of Theorem 1, we have that $E$ is not an eigenvalue of $H$.

In the next section we provide the main technical tool to follow the approach of [2] and then prove Theorem 1$]$

\section{A CRITERION FOR ABSENCE OF DECAYING SOLUTIONS}

We wish to show how local reflection symmetries of the potential can be used to study the behavior of solutions to (4). We follow the approach of [2], which is possible due to our main technical result, Lemma 1 below. Let us remark that once a result like Lemma 1 is established, the remaining part of the proof is quite model independent, so that we could in principle cover more general operators such as 
Schrödinger operators with local point interactions or Sturm-Liouville operators. For reasons of clarity and brevity, we restrict ourselves to the standard Schrödinger case. The reader may convince himself that a similar proof can be given, under suitable assumptions on the involved quantities, for these more general models.

A crucial fact in the discrete case is the special form of the transfer matrix in the presence of a local reflection symmetry. Namely, it was shown in [2] that in this case, $M_{E}(n)$ (with the standard transfer matrix $M_{E}(n)$ associated with a discrete one-dimensional Schrödinger operator) has the form

$$
M_{E}(n)=\left(\begin{array}{rr}
a & -b \\
b & d
\end{array}\right)
$$

with suitable numbers $a, b, d$ which depend on $E$ and $n$. While this is not true in general in the continuum case, we shall show below that the transfer matrix is always conjugate to a matrix of this form, and moreover, the conjugacy is independent of the potential and the energy. This will prove to be sufficient to proceed similarly to [2] in order to prove Theorem [1.

Lemma 1. Suppose that for some $x \in(0, \infty)$, we have $V(y)=V(x-y)$ for every $0 \leq y \leq x / 2$. Then for every $E$, there are numbers $a, b, d$, depending on both $x$ and E, such that

$$
M_{E}(x)=U \cdot\left(\begin{array}{rr}
a & -b \\
b & d
\end{array}\right) \cdot U^{-1}
$$

where

$$
U=U^{-1}=\frac{1}{\sqrt{2}}\left(\begin{array}{rr}
1 & 1 \\
1 & -1
\end{array}\right)
$$

Proof. We begin with a preliminary remark. If $M_{E}(a, b)$ is the transfer matrix for the potential $V$ from $b$ to $a$ and $\tilde{M}_{E}(a, b)$ is the transfer matrix from $b$ to $a$ for the reflected potential $\tilde{V}, \tilde{V}(t)=V(b+a-t)$, then

$$
M_{E}(a, b)=T \cdot \tilde{M}_{E}(a, b)^{-1} \cdot T,
$$

where the matrix $T$ is given by

$$
T=\left(\begin{array}{rr}
1 & 0 \\
0 & -1
\end{array}\right) .
$$

Indeed, $\tilde{u}(t)=u(a+b-t)$ solves $-\tilde{u}^{\prime \prime}+\tilde{V} \tilde{u}=E \tilde{u}$ with initial conditions $\tilde{u}(b)=u(a)$, $\tilde{u}^{\prime}(a)=-u^{\prime}(b)$ and final conditions $\tilde{u}(a)=u(b), \tilde{u}^{\prime}(b)=-u^{\prime}(a)$. Since

$$
\left(\begin{array}{c}
u(b) \\
-u^{\prime}(b)
\end{array}\right)=\left(\begin{array}{c}
\tilde{u}(a) \\
\tilde{u}^{\prime}(a)
\end{array}\right)=\tilde{M}_{E}(a, b)\left(\begin{array}{c}
\tilde{u}(b) \\
\tilde{u}^{\prime}(b)
\end{array}\right)=\tilde{M}_{E}(a, b)\left(\begin{array}{c}
u(a) \\
-u^{\prime}(a)
\end{array}\right),
$$

we get

$$
\left(\begin{array}{c}
u(a) \\
u^{\prime}(a)
\end{array}\right)=\left(\begin{array}{rr}
1 & 0 \\
0 & -1
\end{array}\right) \tilde{M}_{E}(a, b)^{-1}\left(\begin{array}{rr}
1 & 0 \\
0 & -1
\end{array}\right)\left(\begin{array}{c}
u(b) \\
u^{\prime}(b)
\end{array}\right),
$$

which is (7).

We apply this observation to the situation at hand. Since the potential is locally symmetric about the point $x / 2$, we can write the matrix $M_{E}(x)$, using (7), as

$$
M_{E}(x)=T \cdot M_{E}(x / 2)^{-1} \cdot T \cdot M_{E}(x / 2) .
$$


Write

$$
M_{E}(x / 2)=\left(\begin{array}{ll}
\alpha & \beta \\
\gamma & \delta
\end{array}\right)
$$

and compute $M_{E}(x)$ using (8). This gives

$$
M_{E}(x)=\left(\begin{array}{cc}
\alpha \delta+\beta \gamma & 2 \beta \delta \\
2 \alpha \gamma & \alpha \delta+\beta \gamma
\end{array}\right) .
$$

Finally, computing

$$
U^{-1} M_{E}(x) U=\left(\begin{array}{cc}
\alpha \delta+\beta \gamma+\alpha \gamma+\beta \delta & \alpha \gamma-\beta \delta \\
\beta \delta-\alpha \gamma & \alpha \delta+\beta \gamma-\alpha \gamma-\beta \delta
\end{array}\right),
$$

we can conclude with

$$
\begin{aligned}
& a=\alpha \delta+\beta \gamma+\alpha \gamma+\beta \delta, \\
& b=\beta \delta-\alpha \gamma \\
& d=\alpha \delta+\beta \gamma-\alpha \gamma-\beta \delta .
\end{aligned}
$$

We can now prove the following theorem, which is in fact a stronger version of Theorem 1 and whose idea of proof is borrowed from [2].

Theorem 2. Fix some $E \in \mathbb{R}$. Suppose that

(i) there exists a sequence $x_{k} \rightarrow \infty$ such that for every $k$ and every $0 \leq x \leq$ $x_{k} / 2$, we have $V(x)=V\left(x_{k}-x\right)$;

(ii) the sequence $\left(\left|\operatorname{tr} M_{E}\left(x_{k}\right)\right|\right)_{k \in \mathbb{N}}$ does not diverge to $\infty$.

If there exists a nontrivial solution $\phi$ to (4) with $|\phi(x)|^{2}+\left|\phi^{\prime}(x)\right|^{2} \rightarrow 0$ as $x \rightarrow \infty$, we have

(a) the sequence $\left(\left|\operatorname{tr} M_{E}\left(x_{k}\right)\right|\right)_{k \in \mathbb{N}}$ converges to 0 ;

(b) the vector $\left(\phi(0), \phi^{\prime}(0)\right)^{\mathrm{T}}$ is equal to either $U(1,1)^{\mathrm{T}}$ or $U(1,-1)^{\mathrm{T}}$ in $P\left(\mathbb{R}^{2}\right)$.

Proof. According to assumption (i) and Lemma 1 we can define numbers $a_{k}, b_{k}, d_{k}$ by

$$
M_{E}\left(x_{k}\right)=U \cdot\left(\begin{array}{rr}
a_{k} & -b_{k} \\
b_{k} & d_{k}
\end{array}\right) \cdot U^{-1} .
$$

A standard argument (constancy of the Wronskian) gives $\operatorname{det} M_{E}\left(x_{k}\right)=1$ for every $k$; hence

$$
a_{k} d_{k}+b_{k}^{2}=1 \text { for every } k \text {. }
$$

Moreover, by assumption (ii), there is a subsequence of $\left(x_{k}\right)_{k \in \mathbb{N}}$ on which the trace of $M_{E}\left(x_{k}\right)$ is bounded by some constant $C<\infty$, that is,

$$
\left|a_{k_{j}}+d_{k_{j}}\right| \leq C \text { for some sequence } k_{j} \rightarrow \infty .
$$

Assume there is a nontrivial solution $\phi$ to (4) with $|\phi(x)|^{2}+\left|\phi^{\prime}(x)\right|^{2} \rightarrow 0$ as $x \rightarrow \infty$. Write $\Phi(x)=\left(\phi(x), \phi^{\prime}(x)\right)^{\mathrm{T}}$. Clearly,

$$
\Phi\left(x_{k}\right)=M_{E}\left(x_{k}\right) \Phi(0)=U \cdot\left(\begin{array}{rr}
a_{k} & -b_{k} \\
b_{k} & d_{k}
\end{array}\right) \cdot U^{-1} \Phi(0) .
$$

So

$$
\left\|\left(\begin{array}{rr}
a_{k} & -b_{k} \\
b_{k} & d_{k}
\end{array}\right) \Phi\right\| \rightarrow 0 \text { as } k \rightarrow \infty
$$


where $\Phi=U^{-1} \Phi(0)$. From (9) and (11),

$$
\Phi(0)=U \cdot\left(\begin{array}{rr}
a_{k} & -b_{k} \\
b_{k} & d_{k}
\end{array}\right)^{-1} \cdot U^{-1} \Phi\left(x_{k}\right)=U \cdot\left(\begin{array}{rr}
d_{k} & b_{k} \\
-b_{k} & a_{k}
\end{array}\right) \cdot U^{-1} \Phi\left(x_{k}\right) .
$$

This yields

$$
\|\Phi(0)\| \leq\left\|\left(\begin{array}{rr}
d_{k} & b_{k} \\
-b_{k} & a_{k}
\end{array}\right)\right\| \cdot\left\|\Phi\left(x_{k}\right)\right\|,
$$

from which we get (since $\|\Phi(0)\| \neq 0$ and $\|\Phi(x)\| \rightarrow 0$ )

$$
\max \left\{\left|a_{k}\right|,\left|b_{k}\right|,\left|d_{k}\right|\right\} \rightarrow \infty .
$$

This together with (9) and (10) implies

$$
\min \left\{\left|a_{k_{j}}\right|,\left|b_{k_{j}}\right|,\left|d_{k_{j}}\right|\right\} \rightarrow \infty
$$

We see that $\Phi \neq(1,0)^{\mathrm{T}}$ in $P\left(\mathbb{R}^{2}\right)$, for otherwise $a_{k}, b_{k} \rightarrow 0$ and hence, by (9), $d_{k} \rightarrow \infty$, contradicting (10). Similarly, it follows that in $P\left(\mathbb{R}^{2}\right), \Phi \neq(0,1)^{\mathrm{T}}$. Therefore $\Phi=(1, \xi)^{\mathrm{T}}$ in $P\left(\mathbb{R}^{2}\right)$ with some $\xi \neq 0$. Hence, (12) can be rewritten as

$$
a_{k}-\xi b_{k} \rightarrow 0, b_{k}+\xi d_{k} \rightarrow 0 .
$$

Thus,

$$
a_{k}+\xi^{2} d_{k} \rightarrow 0 \text {. }
$$

In particular, we have both $a_{k_{j}}+\xi^{2} d_{k_{j}} \rightarrow 0$ and $\left|a_{k_{j}}+d_{k_{j}}\right| \leq C$, so that (13) implies $\xi^{2}=1$. But then $\operatorname{tr} M_{E}\left(x_{k}\right)=a_{k}+d_{k} \rightarrow 0$, concluding the proof.

Proof of Theorem 1. This is a simple consequence of Theorem 2,

We see that even if we can only prove an upper bound on transfer matrix traces, we can still deduce a result for half-line operators:

Corollary 3 (Half-line case). There are two exceptional boundary conditions $\theta_{1}, \theta_{2}$ such that for every other boundary condition $\theta$, the following holds: Suppose $E$ is such that assumptions (i) and (ii) of Theorem 2 are satisfied. Then $E \notin \sigma_{\mathrm{pp}}\left(H_{\theta}\right)$.

\section{REFERENCES}

[1] D. Damanik, Gordon-type arguments in the spectral theory of one-dimensional quasicrystals, in Directions in Mathematical Quasicrystals, Eds. M. Baake and R. V. Moody, CRM Monograph Series, vol. 13, American Mathematical Society, Providence, RI, 2000, pp. 277-305. MR 2002c: 81048

[2] D. Damanik, J.-M. Ghez, and L. Raymond, A palindromic half-line criterion for absence of eigenvalues and applications to substitution Hamiltonians, Ann. Henri Poincaré 2 (2001), 927-939. MR 2002k:81060

[3] S. Jitomirskaya and B. Simon, Operators with singular continuous spectrum: III. Almost periodic Schrödinger operators, Commun. Math. Phys. 165 (1994), 201-205. MR 97a:47003 
[4] B. Simon, Schrödinger semigroups, Bull. Amer. Math. Soc. 7 (1982), no. 3, 447-526. MR 86b:81001a

Department of Mathematics 253-37, California Institute of Technology, Pasadena, CALifornia 91125

E-mail address: damanik@its.caltech.edu

Department of Mathematics 253-37, California Institute of Technology, Pasadena, CALIFORNia 91125

E-mail address: dirkh@caltech.edu

Current address: Department of Mathematics, University of Illinois at Urbana-Champaign, 1409 W. Green St., Urbana, Illinois 61801

E-mail address: dirk@math.uiuc.edu 